\title{
Liver Vascularity and Function in Fetuses with Trisomy 21
}

\author{
Mona Zvanca, Radu Vladareanu
}

\begin{abstract}
Among the clinical forms of Down syndrome there is a wide spectrum, which includes variable degrees of hematological anomalies. The characteristic type of leukemia for these children is acute megakaryoblastic leukemia (AMKL), which has an incidence 500 times higher in children with Down syndrome compared to normal pediatric population. Between 4 and 10\% of the newborns with Down syndrome will develop a proliferative anomaly of the megakaryoblastic progenitors, named transient myeloproliferative disorder (TMD). Trisomy 21 AMKL presents a series of characteristics which separate it from any other leukemia type within a normal karyotype context.

The anomalies of the hematopoietic system in fetuses with Down syndrome may be suspected either by hematological tests-invasive, only for selected cases, or by ultrasound. Assessing the fetal liver size and investigating the blood flow through the hepatic artery may offer information about hepatic function and hematopoiesis in fetal life.

There are a few studies that have tried to offer an insight on this subject, mainly data on normal growth curves of fetal liver and association beween liver hemodynamics and different pathological situations. Also, there are suggestions about a link between the blood flow in the hepatic artery and fetal aneuploidies and structural malformations.
\end{abstract}

Keywords: Down syndrome, Acute megakaryoblastic leukemia, Transient myeloproliferative disorder, Hepatic artery, Fetal liver size.

How to cite this article: Zvanca M, Vladareanu R. Liver Vascularity and Function in Fetuses with Trisomy 21. Donald School J Ultrasound Obstet Gynecol 2012;6(1):97-103.

Source of support: Nil

Conflict of interest: None declared

\section{INTRODUCTION}

One of the most common problems in fetal medicine field is, still, the antenatal diagnosis of aneuploidies, particularly Down syndrome, which is far from approaching a clinically acceptable solution.

\section{Hematological Anomalies in Fetuses with Down Syndrome}

Among the clinical forms of Down syndrome there is a wide spectrum, which encompasses approximately 80 phenotypes. These include, in variable degrees, neurological alterations, with different degrees of neurodevelopmental delay, craniofacial dysmorfism, gastrointestinal anomalies, congenital cardiac malformations, endocrine dysfunctions, hyperflexibility of the joints, alterations of the immune system function. The hematological system may also be affected, children with Down syndrome may present macrocytosis, alterations in the number of platelets and an increased risk to develop leukemia within the first 5 years of life. ${ }^{1}$ Acute lymphoblastic leukemia (ALL) represents the most common form of leukemia during childhood, with an incidence which is 20 times higher in trisomic children compared to euploid population. However, the characteristic type of leukemia for these children is acute megakaryoblastic leukemia (AMKL), which has an incidence 500 times higher in children with Down syndrome compared to normal pediatric population. ${ }^{2}$ Moreover, it is estimated that between 4 and $10 \%$ of the newborns with Down syndrome will develop a proliferative anomaly of the megakaryoblastic progenitors, named transient myeloproliferative disorder(TMD). This proportion may be as high as $20 \%$, according to different studies. ${ }^{3-5}$ Clinically, the symptoms are minimal, most of the affected children are asymptomatic, but blood counts and smears will always reveal circulating blasts. It may be possible, however, that TMD is underdiagnosed, due to its lack of clinical signs and suspicion, especially that in most cases the natural history is towards remission. Only around $30 \%$ of children with Down syndrome that presented TMD with progress towards leukemia, AMKL. This progression may be overt or more frequently, after an apparent period of remission, which may last 4 months or even years. Very seldom the transient disorder per se may develop into serious complications, even leading to death, mostly due to extensive liver fibrosis, secondary to massive megakaryoblastic infiltration.

Trisomy 21 AMKL presents a series of characteristics which separate it from any other leukemia type within a normal karyotype context. Thus, it always develops within a very narrow time window after birth, the first 4 years of life. Second, almost all cases are preceded by TMD, and the megakaryoblasts in this succesion are morphologically, immunophenotypically and ultrastructurally similar. ${ }^{6}$ Third, AMKL, usually, has a very good prognosis. It also has a series of characteristic karyotype and genetic features. All these data suggest the fact that TMD and AMKL are successive events which have a distinct pathogenetic basis, a common generating mechanism which is different from leukemia in children with normal karyotype.

The genetic features refer to GATA 1 mutation, a gene that encodes a phosphorylated, double zinc finger, DNAbinding transcription factor encoded on the X-chromosome. Recently, acquired somatic mutations in one copy of GATA 1 were demonstrated both in TMD and in DS AMKL, while such mutations were absent in non-DS 
AMKL and in DS acute lymphoid leukemia. ${ }^{7,8}$ Within the proposed model of leukemogenesis, this mutation blocks the cellular differentiation.

However, TMD and AMKL represent two distinct steps and the progression from one to the other requires an additional factor. Initially, it was believed that GATA 1 mutation represents this factor. Later the same mutation was detected in normal karyotype persons which did not develop leukemia. In conclusion, it appears that trisomy 21 in itself may be a leukemogenic factor. The cellular and molecular mechanism that generates the alterations in hematopoiesis is yet unclear. But it is clear that the initial alterations begin very early during ontogenesis.

The anomalies of the hematopoietic activity in fetuses with Down syndrome may be suspected either by hematological tests-invasive, only for selected cases or by ultrasound.

It is important to mention that GATA 1 mutation was detected from intrauterine life. It was present at birth in all cases that will develop TMD and part of the trisomic newborns that have no apparent hematological anomalies.

In a laboratory study by Tunstall-Pedoe et $\mathrm{al}^{9}$ they have demonstrated anomalies of the hematopoietic liver and bone marrow as early as the end of the first trimester of intrauterine life. This study was performed using selected mononucleated cells, which do not present GATA 1 mutations, presumed to appear later in fetal development, even though all these cells were originated from Down syndrome conceptuses. In all the cases, there was an important expansion of the megakaryocyte - erythroid progenitor compartment (55.9\% $\pm 4 \%$ vs $17.1 \% \pm 3 \%$ among CD34+ CD38+ cells; $\mathrm{p}<0.001)$ compared to the other compartments, of common myeloid progenitors $(19.6 \% \pm$ $2 \%$ vs $44.0 \% \pm 7 \% ; \mathrm{p}=0.002)$ and granulocyte and monocyte progenitors $(15.8 \% \pm 4 \%$ vs $34.5 \% \pm 9 \%$; $\mathrm{p}=0.025)$, when compared to specimens from euploid fetuses. These cellular clones present a growth and expansion advantage, but the resulting cells, even though more numerous, are structurally normal.

A vast field for hematological research, all these data suggest that within the spectrum of Down syndrome there are a series of hematological alterations in which TMD and AMKL are only the tip of the iceberg. Most likely there is involvment of several genes and growth factors encoded on chromosome 21 or connected to this. The essential conclusion is that most of the anomalies start developing during the first trimester, a period in which the hematopoiesis is predominantly hepatic. The alterations involve all cases with trisomy 21, not only those that will develop TMD or AMKL.

\section{Measurement of Fetal Liver}

Assessing the size of the fetal liver was a challenging goal from early times of the obstetric ultrasound examination. A large organ, relatively easy to visualize, with important metabolic function and in the first trimester, headquarter of the hematopoietic activity, it benefited from several attempts to standardize measurement criteria. Initially, in 1989, the liver height was measured from the diaphragmatic dome to the inferior border, meaning the height of the right lobe. ${ }^{10}$ One of the main disadvantages is that the method assesses the lobe of lesser importance. The main advantage, however, is the facility of the measurement, especially with older, low resolution machines. Normal growth curves followed, starting from 13 weeks, when the liver height is around 13 $\mathrm{mm}$, until term, when it measures $60 \mathrm{~mm} .^{11}$

In the 1990s with the progress of the sonographic technique and with the event of three-dimensional ultrasound, the possibility of measuring the hepatic volume becomes real. This approach is much more reliable, considering the irregular shape of the organ. Initially, the liver volume could be measured only after 20 weeks, when it is situated around $10 \mathrm{ml}$, with an exponential growth up to $20 \mathrm{ml}$ at 30 weeks and $150 \mathrm{ml}$ at term ${ }^{12,13}$ (Table 1, Fig. 1).
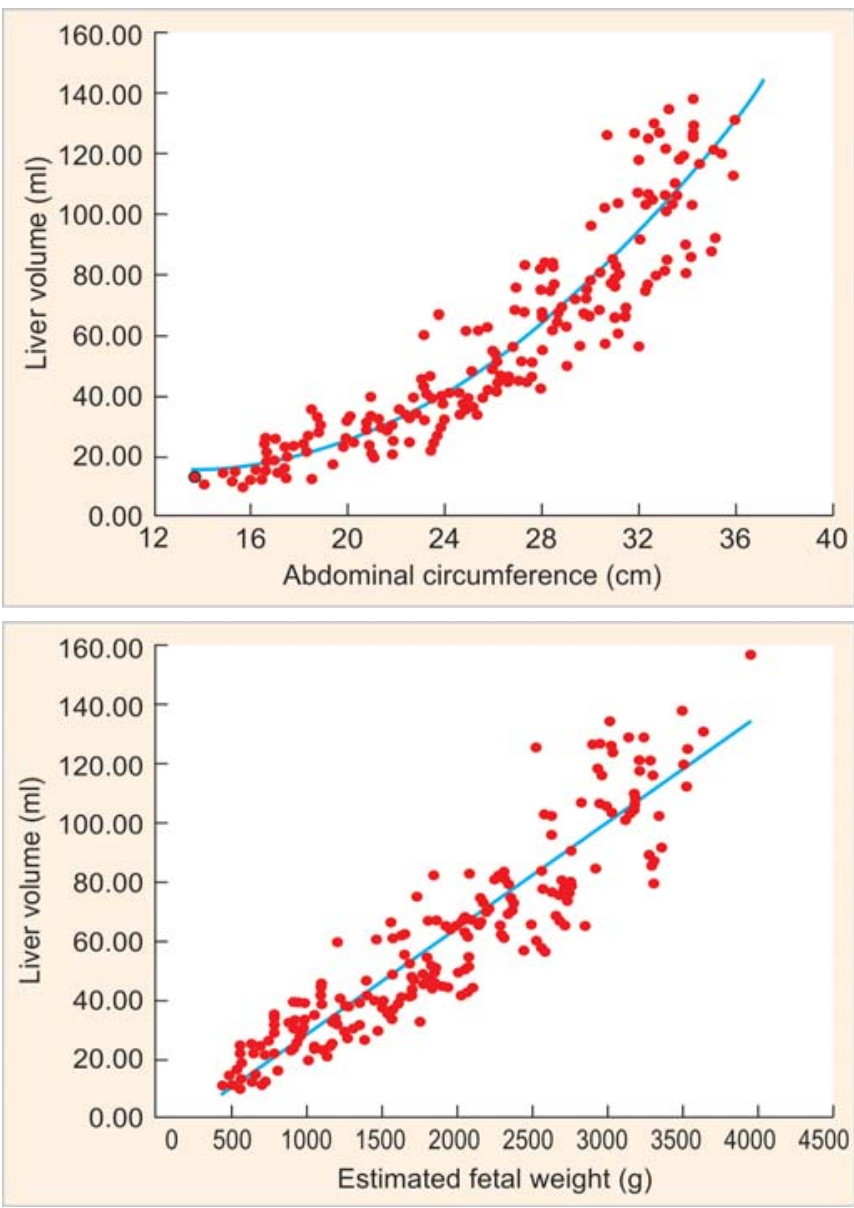

Fig. 1: Liver growth is exponential, in relation to fetal weight and fetal abdominal circumference (Chang et al, 2003) 
Liver Vascularity and Function in Fetuses with Trisomy 21

Table 1: Liver volume according to gestational age and normal growth curves and standard deviations (Chang et al, 2003)

\begin{tabular}{|c|c|c|c|c|c|c|c|c|}
\hline \multirow[b]{2}{*}{$G A$} & \multicolumn{7}{|c|}{ Liver volume $(\mathrm{ml})$} & \multirow[b]{2}{*}{$S D$} \\
\hline & 5 th & 10th & 25 th & 50th & 75 th & 90th & 95th & \\
\hline 20 & 3.09 & 5.05 & 8.26 & 11.84 & 15.42 & 18.63 & 20.59 & 5.31 \\
\hline 21 & 6.66 & 8.71 & 12.05 & 15.77 & 19.50 & 22.84 & 24.88 & 5.52 \\
\hline 22 & 9.70 & 11.82 & 15.29 & 19.17 & 23.04 & 26.51 & 28.64 & 5.74 \\
\hline 23 & 12.34 & 14.54 & 18.14 & 22.16 & 26.18 & 29.79 & 31.99 & 5.95 \\
\hline 24 & 14.72 & 17.00 & 20.74 & 24.50 & 29.07 & 32.80 & 35.09 & 6.17 \\
\hline 25 & 16.99 & 19.35 & 23.22 & 27.53 & 31.84 & 35.70 & 38.07 & 6.39 \\
\hline 26 & 19.28 & 21.73 & 25.72 & 30.18 & 34.64 & 38.63 & 41.07 & 6.60 \\
\hline 27 & 21.74 & 24.27 & 28.39 & 33.00 & 37.60 & 41.73 & 44.25 & 6.82 \\
\hline 28 & 24.52 & 27.32 & 31.38 & 36.13 & 40.88 & 45.13 & 47.74 & 7.04 \\
\hline 29 & 27.74 & 30.42 & 34.81 & 39.71 & 44.60 & 48.99 & 51.67 & 7.25 \\
\hline 30 & 31.56 & 34.32 & 38.84 & 43.88 & 48.92 & 53.44 & 56.20 & 7.47 \\
\hline 31 & 36.11 & 38.95 & 43.60 & 48.79 & 53.98 & 58.63 & 61.47 & 7.69 \\
\hline 32 & 41.53 & 44.46 & 49.24 & 54.57 & 59.91 & 64.69 & 67.61 & 7.90 \\
\hline 33 & 47.98 & 50.98 & 55.89 & 61.37 & 66.85 & 71.77 & 74.77 & 8.12 \\
\hline 34 & 55.58 & 58.67 & 63.71 & 69.34 & 74.96 & 80.00 & 83.09 & 8.33 \\
\hline 35 & 64.49 & 67.65 & 72.83 & 78.60 & 84.37 & 89.54 & 92.71 & 8.55 \\
\hline 36 & 74.84 & 78.08 & 83.38 & 89.30 & 95.22 & 100.53 & 103.77 & 8.77 \\
\hline 37 & 86.77 & 90.09 & 95.53 & 101.59 & 107.66 & 113.09 & 116.42 & 8.98 \\
\hline 38 & 100.43 & 103.83 & 109.40 & 115.61 & 121.82 & 127.38 & 130.79 & 9.20 \\
\hline 39 & 115.95 & 119.44 & 125.13 & 131.49 & 137.85 & 143.54 & 147.03 & 9.42 \\
\hline 40 & 133.49 & 137.05 & 142.88 & 149.38 & 155.88 & 161.71 & 165.27 & 9.63 \\
\hline
\end{tabular}

Researchwise, it is important to mention that these volumes are aquired using two-dimensional ultrasound, by measuring the three dimensions of the fetal liver and calculating the volume according to a mathematical formula. Thus, the measurements may be different when using ultrasound volumetric techniques (VOCAL, multiplanar).

Measurement of the fetal liver faced a series of difficulties over time. Initially, it was the lack of an adecquate tool. This was overcome with the appearance of the VOCAL software - semiautomatic volume measurement of structures with irregular shape and different echogenicities. Fetal liver, however, as an abdominal organ with an echostructure that is only slightly different from the neighboring organs, may be very difficult to visualize and measure, especially in the posterior area, where it may be shadowed by ribs and spine. This shadowing becomes more intense with advancing gestational age. During the first trimester the main limitation is related to fetal size, which may be overcome only by using a high performance, high resolution ultrasound machine in order to differentiate between structures that are only a few millimeters in size.

The most recent study regarding fetal liver size comes from Dos Santos Rizzi et al 2010, which, by using the multiplanar technique, between 27 and 38 weeks proved a very good intra- and interobserver reproducibility measurement technique with a confidence interval of 0.993 and 0.984 respectively. On the other hand this technique has a limited clinical application, because it requires a large number of sections and serial measurements, which is timeconsuming. ${ }^{14}$

\section{Liver Hemodynamics: The Role of Ductus Venosus}

Partly, the interest for measuring the fetal liver is related to the hepatic blood flow regulation, which, apparently, places the liver as a preferentially spared organ, according to several studies by Kilavuz. ${ }^{15}$ Even when the umbilical blood flow is decreased and a large amount of blood is shunted through ductus venosus, the intrahepatic amount of blood is maintained. This is the consequence of activating a secondary blood supply, the hepatic artery.

In midpregnancy, under physiological circumstances, approximately $30 \%$ of the umbilical flow is shunted through ductus venosus toward the heart and systemic circulation, especially brain. After 30 weeks, this fraction decreases to $20 \%$ and it will be maintained, so throughout the rest of the pregnancy, the remaining 70 to $80 \%$ of the umbilical venous, flow is distributed towards the liver. Hence, the metabolic importance of the liver, especially the left lobe, which receives the largest amount of the umbilical blood supply. The right lobe, less important in fetal life, following the distribution of the portal vessels, receives mainly venous blood from the splanchnic territory. Postnatally, with the event of neonatal digestion, the right lobe gains a more prominent role, increases in size, surpassing the left lobe, which after birth loses its main blood source.

The ductus venosus represents much more than a simple vascular channel. Its size is sensitive to adrenergic stimulation, prostaglandins and nitric oxide, all of these substances acting as vasoconstrictors. The strongest vasodilator is hypoxemia, which may increase the vascular 
diameter by $60 \%$. Even though the isthmus has the most prominent muscular layer, the changes in caliber extend to the full length of the vessel, which has an important impact on the flow speed and resistance and pulsatility indices.

Equally important in regulating the blood flow through the ductus venosus are the rheological parameters, viscosity and blood pressure. The blood in the ductus venosus is characterized by high velocity and low viscosity. In contrast, the hepatic tissue represents a very large capillary surface, in which the blood travels with very low speed. At low velocities, the blood loses its newtonian characteristics, the viscosity increases and so does the resistance index, and closing pressure is 1 to $4 \mathrm{~mm} \mathrm{Hg}$. Therefore, even a minimal increase in hematocrit has a very high impact, with a drastic increase in viscosity and reduction of the flow in the hepatic capillaries. ${ }^{16,17}$

The reduced proportion of shunting through the ductus venosus in the last 8 to 10 weeks implies an increase in the blood is distributed to the liver. Therefore, it is suggested that there is a priority in the development and the function of the liver at this time of pregnancy.

Under hypoxic conditions, these priorities change. More blood is shunted through the ductus venosus toward heart, brain, adrenals, a situation that may prolong indefinitely. Obviously, in these conditions, the hepatic tissue will be deprived by oxygen, which may alter its long-term development. Also, the degree of oxygen extraction by the hepatocytes is quite low, only 10 to $15 \%$.

\section{The Hepatic Artery and Liver Hemodynamics}

Besides the umbilical vein, which is the most important contributor to intrahepatic blood flow, the liver has two additional blood sources. The venous portal system, which connects to the umbilical vein through the left portal vein, is responsible for a supplementary $20 \%$, a poorly oxygenated blood and with little nutrients. The third source is the hepatic artery, a small size vessel, with, apparently, a minor functional importance. It supplements up to $10 \%$ of the hepatic blood flow under physiological conditions (Figs 2 and 3).

Even though the hepatic artery has a relatively modest contribution to fetal hepatic vascularity, its role in adult life is important. The hemodynamic model known as buffer system was described initially in patients with cirrhosis. This implies that the caliber of the hepatic artery, and hence, the blood flow through the hepatic artery increases when inflow through other vascular sources of the liver is diminished. The increase in diameter is mediated through adenosinereceptors which may be found lining the hepatic artery wall. Uteroplacental insufficiency or other cause of fetal distress,

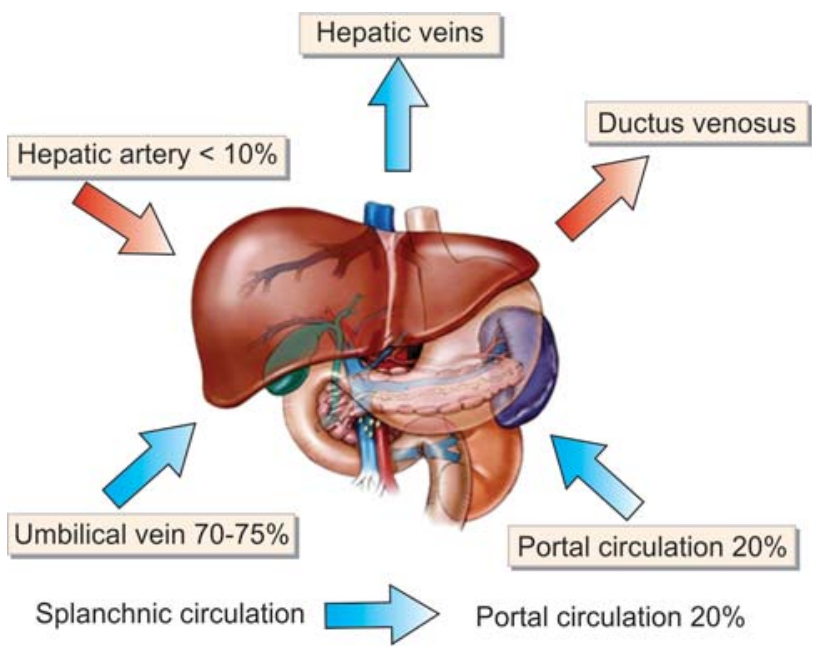

Fig. 2: The schematic representation of the hepatic hemodynamics

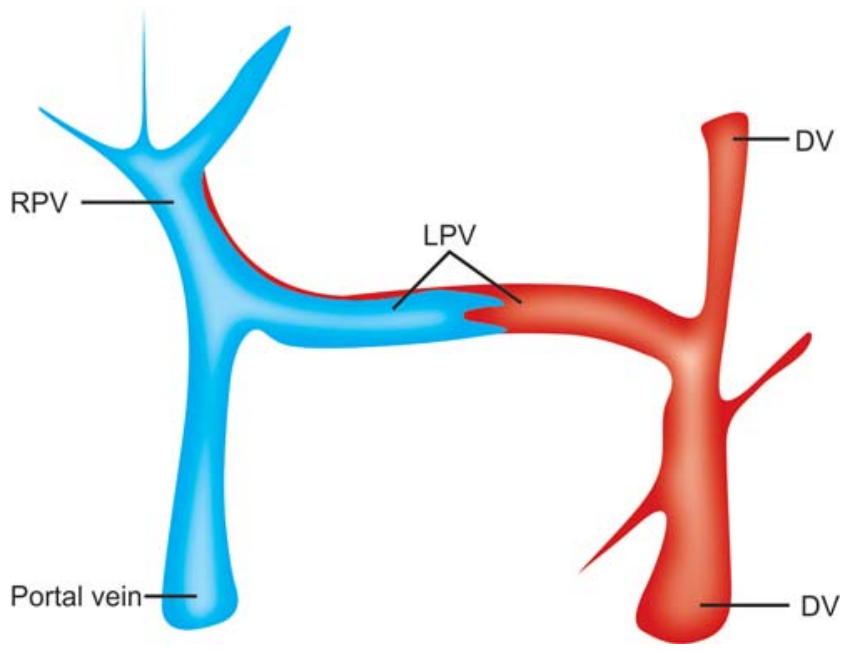

Fig. 3: Schematic representation of the main blood distribution toward right and left liver lobes (RPV: right portal vein; LPV: left portal vein; DV: ductus venosus; UV: umbilical vein)

with a reduction in umbilical vein flow is followed by increased shunting through ductus venosus and a reduction of the blood flow to the liver. There is reduced clearance of the adenosine and the increased concentration acts on the hepatic artery as a vasodilator, thus maintaining the intrahepatic blood flow. The mechanism is very prompt and detectable even in very drastic reductions of the umbilical and splanchnic flows.

Starting from the initial model, this mechanism was studied in fetal life and a series of well-documented studies followed.

Ebbing in $2008^{18}$ described the hemodynamic parameters of the hepatic artery in three cases : Fetal nonimmune anemia, intrauterine growth restriction, acute posthemorrhagic maternal anemia. These cases were compared with results from 161 low-risk pregnancies. This was considered a pilot study, investigating measurement criteria 
and normal values. Gestational age at enrollment was between 19 and 28 weeks, with a median of 23 weeks. Each case within the control group was examined 3 times, at 3 to 5 weeks interval. The hepatic artery was visualized in any of the transverse, oblique and sagittal planes, depending on the examination conditions and the best measurement was kept for record. Peak systolic velocity (PSV) and pulsatility index (PI) were recorded and data was compared to the systemic circulation by using the hemodynamic parameters of the umbilical and middle cerebral artery.

A subsequent study from the same team, ${ }^{19}$ evaluates the blood flow in the hepatic artery in fetuses with growth restriction, starting from the normal range described above. This second study presents a new concept of the role of splenic and superior mesenteric artery in regulating liver hemodynamics. The splenic artery may influence the intrahepatic venous portal flow. The pulsatility index of the splenic artery is reduced, whenever, the umbilical venous blood flow is reduced, with reduction in the right hepatic lobe perfusion. A similar effect, even though of lesser importance is expected to be seen in the superior mesenteric artery.

The hepatic artery was evaluated in sagittal or transverse section of the fetal abdomen. The left branch of the hepatic artery is more accesible to measurement, neighboring the ductus venosus. The splenic artery was identified in axial section, at its origin from celiac trunk, in front of the descending aorta and posterior to the stomach (Fig. 4). The superior mesenteric artery represents the second vessel originating from the subdiaphragmatic descending aorta, inferior and close to the celiac trunk, visible, also, in sagittal section (Fig. 5).

In cases with intrauterine growth restriction the PI of the splenic and hepatic artery was found to be reduced, according to what was already suspected. However, the physiopathological mechanism is not completely understood.

Unlike was initially expected, the superior mesenteric artery did not present any functional changes, probably due to its distant position or to a late intervention.

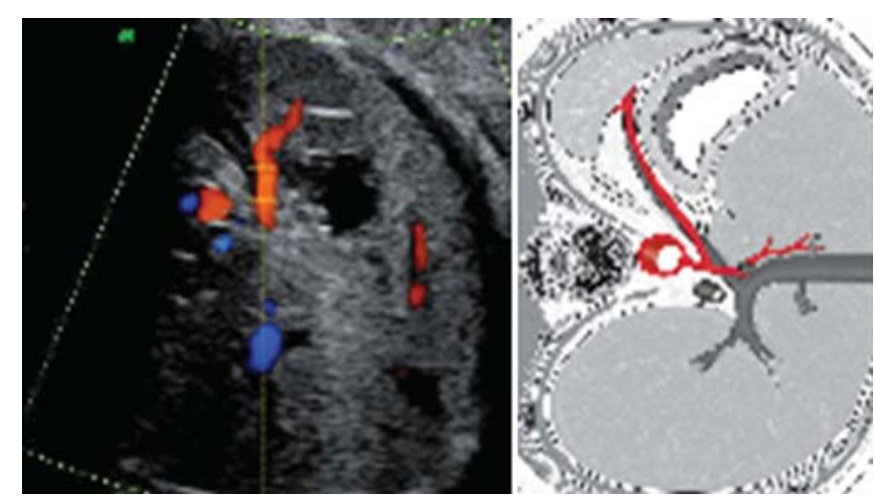

Fig. 4: Splenic artery in transverse section of the fetal abdomen
Measurements were possible in all cases with intrauterine growth restriction. The brain-sparing effect, associated with splanchnic vasoconstriction was reflected as vasodilation of the hepatic and splenic artery, with low PI. This suggests a direct hepatic arterial contribution to the sinusoidal flow and a significant splenic contribution to sinusoidal portal flow whenever, the umbilical inflow toward right hepatic lobe is reduced. These changes are aimed to adapt the fetus to hypoxemic conditions and ensure neurological protection and short-term survival, but may have long-term consequences over hepatic and general metabolic function of the individual.
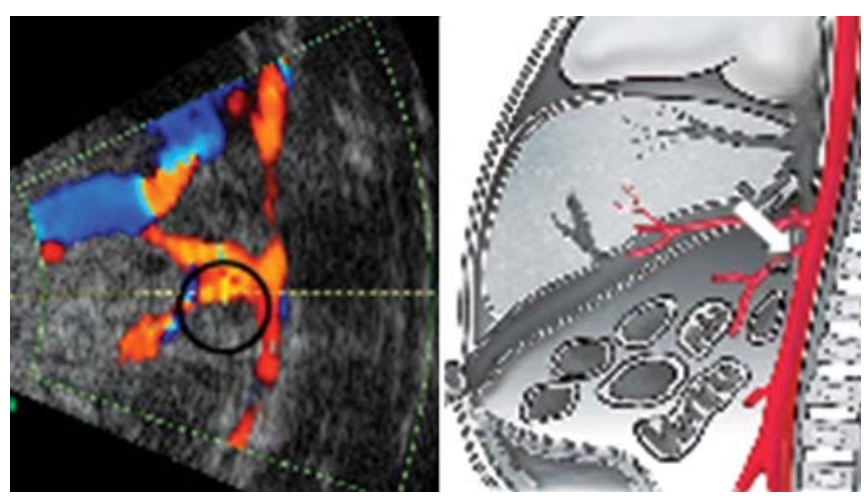

Fig. 5: Superior mesenteric artery, the second vessel originating from the subdiaphragmatic descending aorta, in sagittal section of the fetal abdomen

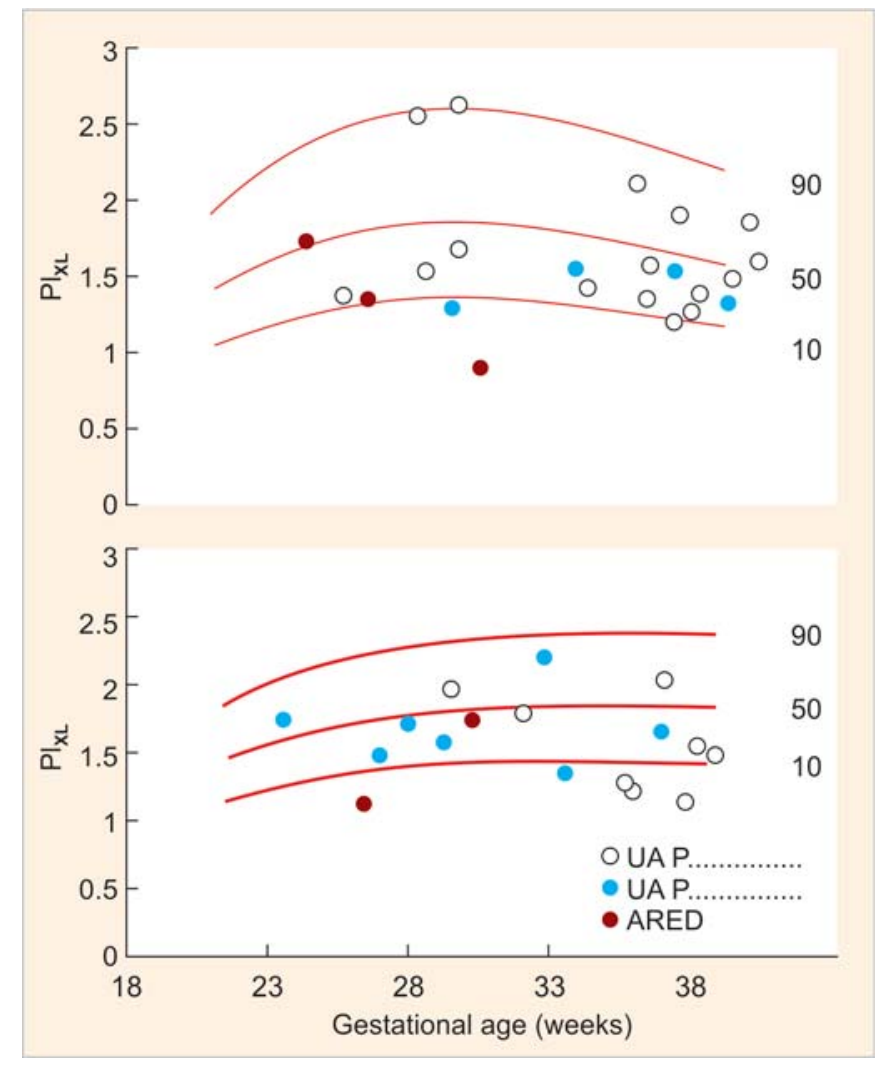

Fig. 6: Variation in hepatic artery and splenic artery PI in fetuses with IUGR, compared to PI values of the umbilical 
The adaptative changes do not seem to progress with increasing PI values of the umbilical artery. The only differences are related to gestational age at the onset of IUGR (Fig. 6). Thus, the earlier the fetal growth is affected, the more important the arterial hepatic vasodilation. It may be concluded that the PI values reflect the degree of fetal compromise.

This study does not investigate and offer any data regarding the velocity through any of the studied vessels.

One of the most recent publications regarding the hemodynamics of the hepatic artery comes from Bilardo team. ${ }^{20}$ This study focuses on differences in hepatic artery blood flow in fetuses with aneuploidies or structural malformations at the end of the first trimester. All cases included in this study were 11 to 14 weeks pregnancies, with a high risk for aneuploidies, with severe sonographic anomalies, hydrops or increased nuchal translucency. The hepatic arterial signal was particularly strong in these cases. It was a prospectively organized study, with assessment of the pulsatility indices of the hepatic artery in fetuses with increased risk for aneuploidies. There is also an attempt to demonstrate the physiopathological background for the Doppler changes in these fetuses. All measurements could be completed in 59 fetuses, including crown-rump length, nuchal translucency, assessment of the nasal bone, blood flow through ductus venosus and across tricuspid valve, detailed anatomical survey and PI of the hepatic artery (HA). In all cases the karyotype was known, as the ultrasound was followed by chorionic villus sampling.

The flow in the hepatic artery was assessed using the same section and conditions as for measurement of the blood flow through ductus venosus. After measuring the last one, the gate was slightly displaced inferiorly, toward descending aorta, where a small vessel is seen, close to ductus venosus depending on the position and the size of the pulsed wave gate the hepatic artery, or the ductus venosus or both may be seen, on different channels. After recording at least three consecutive waves, the PI of the hepatic artery was measured using the automated system.

In fetuses with normal NT and normal outcome the mean HA PI was 2.03 (SD 0.46). HA PI was significantly lower (independent sample t-tests, $\mathrm{p}<0.05$ ) in fetuses with enlarged NT (1.76, SD 0.37), chromosomal anomalies (all: 1.60, SD 0.28; Down syndrome: 1.57, SD 0.26) and in chromosomally normal fetuses with other causes of adverse outcome (1.67, SD 0.27). Fetuses with chromosomal anomalies have low mean HA PI, irrespective of the degree of NT enlargement and of gestational age. In fetuses with enlarged NT there was a significant correlation between NT and DVPIV (Spearman rho 0.30) and a significant negative correlation between HA PI and NT (Spearman rho $=0,34$ ) and HAPI and DVPIV (Spearman rho $=0.58$ ).

This study is important because it is the first one to investigate the liver hemodynamic during the first trimester, starting from the basic knowledge from second and third trimesters and postnatal life. Moreover, it suggests that the hepatic artery may be important in a context other than maintaining the intrahepatic blood volume. The changes in hepatic artery blood flow in association with aneuploidies and other genetic syndromes and fetal structural anomalies are confusing, especially when considering that many of these situations are not likely to be physiopathologically related. The inverse relationship between the PI in the hepatic artery and PI in ductus venosus is consistent with results from previous studies, which demonstrate dilatation of the hepatic artery (with a decrease in PI) whenever, there is an increase in ductus venosus shunting. Ductus venosus, due to its anatomy — short vessel, small caliber, communicating with large vascular systems at both extremitiesunlike other blood vessels, is characterized by a high PI whenever, the blood flow through the vessel is increased. The cardiac dysfunction may be a common nominator between the modified ductus venosus flow and aneuploidy.

One of the physiopathological theories is based on the adenosine-mediated mechanism. Low resistance to flow in the hepatic artery may be the result of the endothelial response to vasoactive substances, a reaction that is suggested to be present from the end of the first trimester. This study does not measure velocities in the hepatic artery, but implies that these are incresed in fetuses with low arterial resistance. On the other hand, it is not obvious why chromosomal anomalies would alter the venous blood flow toward the liver, in order to require arterial compensation.

Another hypothesis, according to Bilardo, may be centered on the hematopoietic mechanism, similar to cases of anemia, or hepatopoietic mechanism, as in cases of hypoxia-induced liver dysfunction. However, none of these mechanisms are proved to be active during the first trimester, even though this may be possible.

The increase in the vascular flow in fetuses with trisomy 21 may, also, be the result of increased hematopoietic activity in the liver. This is consistent with previous animal studies which suggest that hepatic hematopoiesis is modulated by the arterial blood flow. ${ }^{21}$

As for hypoxia, it may be responsible for a series of hemodynamic alterations during the first trimester, such as the flow in ductus venosus, right ventricular diastolic dysfunction, discordant ventricular afterload. These are adaptative reactions to low umbilical flow in order to maintain the cerebral perfusion. It is not clear, however, if 
fetuses with trisomy 21 are hypoxic and if so, what is the underlying mechanism. Therefore, the hypoxic theory, even though possible is, yet, unsufficiently demonstrated.

The liver has an important role in regulating fetal growth, progressing from a mainly hematopoietic function during the first trimester, to a hepatopoietic function during the second and third trimesters. Hepatocyte differentiation is essentially related to signals from the extracellular matrix, soluble factors from the surrounding cells or to direct cellular interaction. The hepatic function was proved to be altered in fetuses with chromosomal aberrations during the second and third trimesters as well as postnatally. This alteration is translated through low levels of alfa-fetoprotein, associated with different hematological disorders, more or less clinically obvious. In this context the increase in hepatic arterial blood flow may be an attempt to stabilize the intrahepatic perfusion and hence, the hepatocyte function.

\section{CONCLUSION}

There are very few studies focused on liver function and hemodynamics during fetal life. The interest started only after the description of ductus venosus and with the surprising discovery that liver is a spared organ during fetal distress. The relationship between Down syndrome and liver vascularity is a challenging subject. Even though we are very far from offering a plausible physiopathological explanation, as we are very far from understanding many of the alterations that are associated with this chromosomal disorders, assessing the blood flow through hepatic artery during the first trimester may prove to be a useful tool in raising suspicion of the genetic disease.

\section{REFERENCES}

1. Roizen NJ, Amarose AP. Hematologic abnormalities in children with Down syndrome. Am J Med Genet 1993;46:510-12.

2. Lange B. The management of neoplastic disorders of hematopoiesis in children with Down's syndrome. Br J Hematol 2000;110:512-24.

3. Zipursky A, Brown E, Christensen H, Sutherland R, Doyle J. Leukemia and/or myeloproliferative syndrome in neonates with Down syndrome. Semin Perinatol 1997.

4. Zipursky A. Transient leukaemia: A benign form of leukaemia in newborn infants with trisomy 21. Br J Haematol 2003;120:930.

5. Smrcek JM, Baschat AA, Germer U, Gloeckner-Hofmann K, Gembruch U. Fetal hydrops and hepatosplenomegaly in the second half of pregnancy: A sign of myeloproliferative disorder in fetuses with trisomy 21. Ultrasound Obstet Gynecol 2001;17:403-09.

6. Karandikar NJ, Aquino DB, McKenna RW, Kroft SH. Transient myeloproliferative disorder and acute myeloid leukemia in Down syndrome: An immunophenotypic analysis. Am J Clin Pathol 2001;116:204-10.
7. Wechsler J, Greene M, McDevitt MA, et al. Acquired mutations in GATA 1 in the megakaryoblastic leukemia of Down syndrome. Nat Genet 2002;32:148-52.

8. Hitzler JK, Cheung J, Li Y, Scherer SW, Zipursky A. GATA 1 mutations in transient leukemia and acute megakaryoblastic leukemia of Down syndrome. Blood 2003;101:4301-04.

9. Tunstall-Pedoe O, Roy A, Karadimitris A, de la Fuente J, Fisk $\mathrm{NM}$, et al. Abnormalities in the myeloid progenitor compartment in Down syndrome fetal liver precede acquisition of GATA 1 mutations. Blood 2008;112:4507-11.

10. Roberts AB, Mitchell JM, Pattison NS. Fetal liver length in normal and isoimmunized pregnancies. Am J Obstet Gynecol 1989;161:42-46.

11. Phatihattakorn C, Ruangvutilert P, Sansaneevithayakul P, Boriboonhirunsarn D. Reference centile chart for fetal liver length of Thai fetuses. J Med Assoc Thai 2004;87:750-54.

12. Chang FM, Hsu KF, Ko HC, Yao BL, Chang CH, et al. Threedimensional ultrasound assessment of fetal liver volume in normal pregnancy: A comparison of reproducibility with twodimensional ultrasound and a search for a volume constant. Ultrasound Med Biol 1997;23:381-89.

13. Chang $\mathrm{CH}, \mathrm{Yu} \mathrm{CH}$, Chang FM, Ko HC, Chen HY. The assessment of normal fetal liver volume by three-dimensional ultrasound. Ultrasound Med Biol 2003;29:1123-29.

14. Dos Santos Rizzi MC, Júnior EA, Nardozza LM, Diniz AL, Rolo LC, Moron AF. Nomogram of fetal liver volume by threedimensional ultrasonography at 27 to 38 weeks of pregnancy using a new multiplanar technique. Am J Perinatol 2 Mar, 2010.

15. Kilavuz O, Vetter K. Is the liver of the fetus the 4th preferential organ for arterial blood supply besides brain, heart and adrenal glands? J Perinat Med 1999;27:103-06.

16. Kiserud T. The ductus venosus. Semin Perinatol 2001;25:11.

17. Kiserud T, Acharya G. The fetal circulation. Prenat Diagn 2004;24:1049.

18. Ebbing C, Rasmussen S, Godfrey KM, Hanson MA, Kiserud T. Hepatic artery hemodynamics suggest operation of a buffer response in the human fetus. Reprod Sci 2008 Feb;15(2): 166-78.

19. Ebbing C, Rasmussen S, Godfrey K, Hanson M, Kiserud T. Redistribution pattern of fetal liver circulation in intrauterine growth restriction. Acta Obstetricia et Gynecologica 2009;88:1118-23.

20. Bilardo CM, Timmerman E, Robles de Medina PG, Clur SA. Increased hepatic artery flow in first trimester fetuses: An ominous sign. Ultrasound Obstet Gynecol 2010.

21. Kunisaki SM, Azpurua H, Fuchs JR, Graves SC, Zurakowski D, Fauza DO. Fetal hepatic haematopoiesis is modulated by arterial blood flow to the liver. Br J Haematol Aug 2006; 134(3):330-32.

\section{ABOUT THE AUTHORS}

\section{Mona Zvanca (Corresponding Author)}

Assistant Professor, Obstetrics and Gynecology Clinic, Elias University Hospital, University of Medicine and Pharmacy, Carol Davila, Bucharest Romania, Phone: +40-740956053, e-mail: monazvanca@yahoo.com

\section{Radu Vladareanu}

Professor, Obstetrics and Gynecology Clinic, Elias University Hospital, University of Medicine and Pharmacy, Carol Davila Bucharest, Romania 